\title{
Association of four genetic polymorphisms in the vascular endothelial growth factor-A gene and development of ovarian cancer: a meta-analysis
}

\author{
Chao-Huan $X u^{1}$, Zhong-Hui $\mathrm{He}^{1}$ and Hong $X \mathbf{u}^{1}$ \\ ${ }^{1}$ Department of Gynaecology, First Affiliated Hospital of Guangxi Medical University, Nanning 530021, China \\ Correspondence to: Hong Xu, email: nnxuhong@163.com \\ Keywords: vascular endothelial growth factor, polymorphism, ovarian cancer, meta-analysis
}

Received: May 14, 2017 Accepted: August 09, $2017 \quad$ Published: August 21, 2017

Copyright: Xu et al. This is an open-access article distributed under the terms of the Creative Commons Attribution License 3.0 (CC BY 3.0), which permits unrestricted use, distribution, and reproduction in any medium, provided the original author and source are credited.

\section{ABSTRACT}

This study meta-analyzed the literature on possible association of four polymorphisms $(+936 \mathrm{C} / \mathrm{T},-460 \mathrm{C} / \mathrm{T},-2578 \mathrm{C} / \mathrm{A}$ and $-1154 \mathrm{G} / \mathrm{A})$ in the vascular endothelial growth factor (VEGF)-A gene with risk of ovarian cancer. Meta-analysis of 7 case-control studies involving $+936 \mathrm{C} / \mathrm{T}$, 4 studies involving $-460 \mathrm{C} / \mathrm{T}, 4$ studies involving $-2578 \mathrm{C} / \mathrm{A}$ and 2 studies involving $-1154 \mathrm{G} / \mathrm{A}$ showed significant association between $-460 \mathrm{C} / \mathrm{T}$ and ovarian cancer risk. This risk was observed in the total population (allelic model, OR 1.80, 95\% CI 1.26-2.59, $P=0.001$; recessive model, OR 1.84, 95\% CI 1.13-2.98, $P=0.01$; dominant model, OR 0.51, 95\% CI 0.39-0.67, $P<0.001$; homozygous model, OR 2.48, 95\% CI 1.72-3.56, $P<0.001$; heterozygous model, OR 1.67, 95\% CI 1.26-2.21, $P<0.001$ ) and in the subgroup of Asian study participants. The CA genotype at $\mathbf{- 2 5 7 8 C}$ / A was a risk factor in the total population, while the CT genotype at $+936 \mathrm{C} / \mathrm{T}$ was a protective factor in Caucasians. None of the five genetic models suggested a significant association between $-1154 \mathrm{G} / \mathrm{A}$ and ovarian cancer risk in the entire study population, or between $+936 \mathrm{C} / \mathrm{T}$ and risk in Asian or Chinese participants. These findings should be verified in large, well-designed studies.

\section{INTRODUCTION}

Ovarian cancer is a major cause of cancer-related death in females worldwide $[1,2]$. Although treatment can significantly improve quality of life, the 5-year survival rate for patients with advanced ovarian cancer remains below $30 \%$, mainly due to high rates of recurrence and metastasis [3, 4]. Development of ovarian cancer has been linked to numerous environmental and lifestyle factors, including age, early menarche, late menopause, non-childbearing, high-fat diet, exposure to talcum powder and asbestos, and long-term hormone supplementation [5, 6]. Ovarian cancer has also been linked to several genetic polymorphisms [7-9].

Angiogenesis, which refers to the formation of new capillary blood vessels from preexisting ones, is an important factor in the development and spread of cancer, including ovarian cancer [10-12]. A key mediator of angiogenesis is vascular endothelial growth factors (VEGFs) [13], which are expressed at higher levels in malignant ovarian tumor tissues than in benign tumor tissues or tissue of low malignant potential [14-16]. This implicates VEGFs in the pathological angiogenesis of ovarian cancer. Indeed, prognosis and overall survival of ovarian cancer patients correlate with serum and/or tumor levels of VEGFs [17-21]. These findings suggest that genetic factors affecting VEGF expression or activity may influence ovarian cancer development and progression.

The founding member of the VEGF family, VEGF-A, is encoded by a gene on chromosome $6 \mathrm{p} 12$ that comprises a $14-\mathrm{kb}$ coding region of eight exons and exhibits alternate splicing to form a family of proteins. [22]. Several single-nucleotide polymorphisms (SNPs) in this gene correlate with VEGF expression [23-25]. Numerous case-control studies [26-32] have investigated whether polymorphisms in the VEGF-A gene at positions $+936 \mathrm{C} / \mathrm{T}$ (rs3025039), -460C/T (rs833061), -2578C/ A (rs699947) or -1154G/A (rs1570360) influence ovarian cancer risk. Results have been inconclusive and contradictory, prompting us to perform this comprehensive 
meta-analysis of all available evidence on these potential associations. To the best of our knowledge, this is the first meta-analysis concerning all four of these previously analyzed polymorphisms and ovarian cancer risk.

\section{RESULTS}

\section{Description of studies}

A total of 104 potentially relevant publications published in English or Chinese up to April 12, 2017 were systematically identified in PubMed, EMBASE, Google Scholar and Chinese National Knowledge Infrastructure databases (Figure 1). We excluded 83 studies based on review of the titles and abstracts, because they did not analyze the target polymorphisms in the VEGF-A gene or because they did not examine ovarian cancer risk. We excluded another 8 studies because they were not casecontrol studies, 3 studies because they were review articles and 1 study because it did not report precise genotypes. Another 2 studies were excluded because they analyzed overlapping patient populations. In the end, 7 studies were included in the final meta-analysis [26-32] (Table 1).

All 7 studies evaluated the association between the $+936 \mathrm{C} / \mathrm{T}$ polymorphism and ovarian cancer risk (1,345 cases and 1,671 controls). Four studies [26, 29, 31, 32] evaluated the association between the $-460 \mathrm{C} / \mathrm{T}$ polymorphism and ovarian cancer risk ( 813 cases and 905 controls); 4 studies $[28,29,31,32]$, the association between the $-2578 \mathrm{C} / \mathrm{A}$ polymorphism and ovarian cancer risk (1,022 cases and 1,228 controls); and 2 studies [29, 31], the association between the $-2578 \mathrm{C} / \mathrm{A}$ polymorphism and ovarian cancer risk (602 cases and 623 controls). The distribution of genotypes in controls was consistent with Hardy-Weinberg equilibrium (HWE, $P>0.05$ ) in all but one study [32] involving the $-460 \mathrm{C} / \mathrm{T}$ polymorphism.

All studies in the meta-analysis received a score of at least 6 on the Newcastle-Ottawa Scale [34], indicating that they were all of good quality. The mean score for all included studies was 7 (Table 2).

\section{Meta-analysis of studies on the $+936 \mathrm{C} / \mathrm{T}$ (rs3025039) polymorphism}

Meta-analysis of a possible association between $+936 \mathrm{C} / \mathrm{T}$ polymorphism and ovarian cancer risk is summarized in Table 3. Based on the total study population involving 1,345 cases and 1,751 controls, none of the five genetic models indicated a significant association: allelic model, OR 1.17, 95\% CI 0.79-1.72, $P=0.44$ (Figure 2A); recessive model, OR 1.25, 95\% CI $0.82-1.88, P=0.30$ (Figure 2B); dominant model, OR $0.89,95 \%$ CI $0.55-1.45, P=0.65$ (Figure 2C); homozygous model, OR $1.24,95 \%$ CI $0.76-2.03, P=0.39$

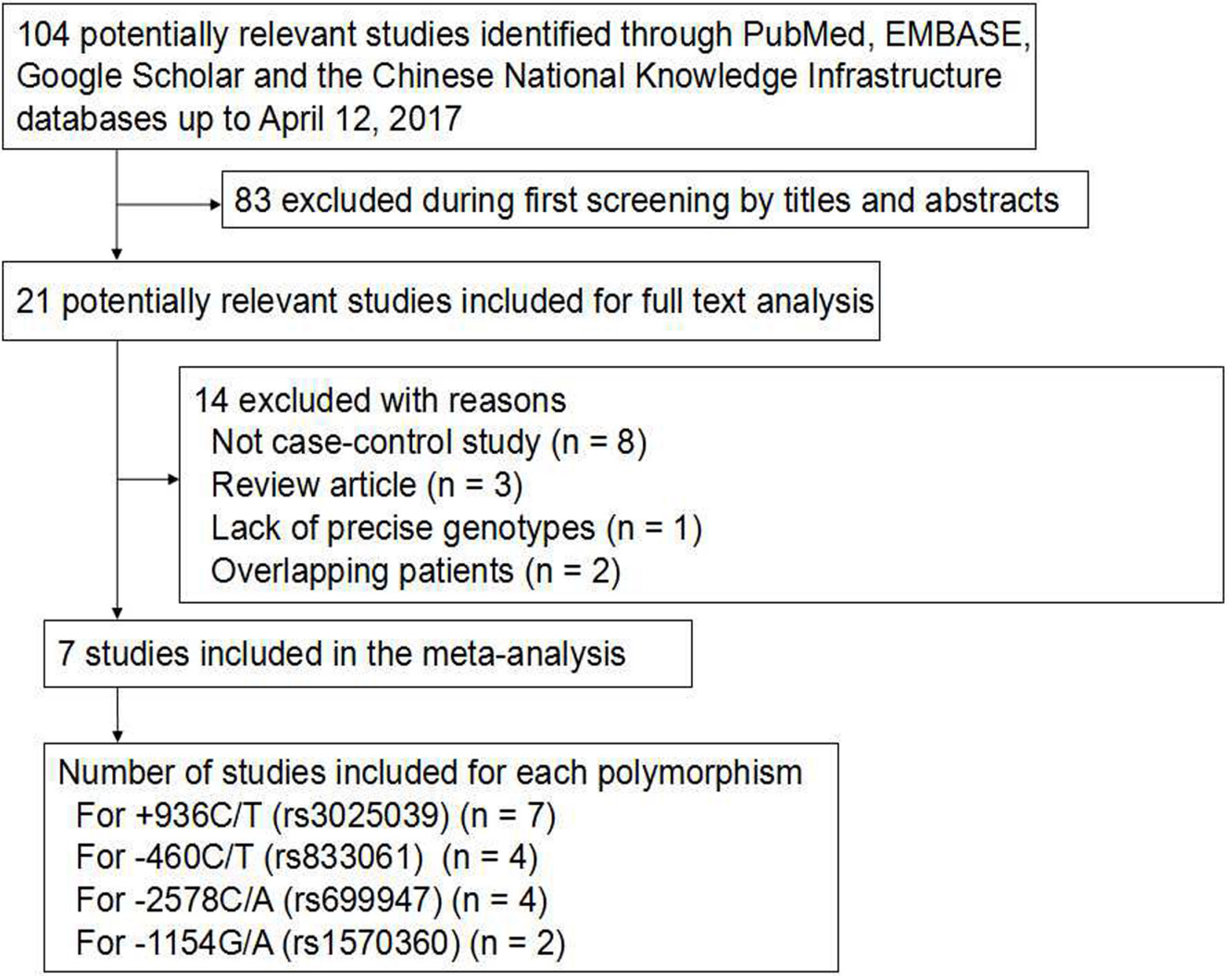

Figure 1: Flowchart of study selection. 


\section{Table 1: Characteristics of studies in the meta-analysis}

\begin{tabular}{|c|c|c|c|c|c|c|c|c|c|c|c|c|c|c|c|c|c|}
\hline \multirow{2}{*}{$\begin{array}{l}\text { First author } \\
+936 \mathrm{C} / \mathrm{T}(\mathrm{rs} 30\end{array}$} & \multirow{2}{*}{$\begin{array}{c}\text { Year } \\
25039)\end{array}$} & \multirow[t]{2}{*}{ Ethnicity } & \multirow[t]{2}{*}{ Country } & \multirow[t]{2}{*}{$\begin{array}{l}\text { Genotyping } \\
\text { method }\end{array}$} & \multirow[t]{2}{*}{$\begin{array}{l}\text { Type of } \\
\text { controls }\end{array}$} & \multirow[t]{2}{*}{$\begin{array}{l}P \text { for } \\
\text { HWE }\end{array}$} & \multirow[t]{2}{*}{$\begin{array}{l}\text { Cases/ } \\
\text { Controls }\end{array}$} & \multicolumn{3}{|c|}{ No. of cases } & \multicolumn{2}{|c|}{$\begin{array}{l}\text { Allele frequencies of } \\
\text { cases, } n,(\%)\end{array}$} & \multicolumn{3}{|c|}{ No. of controls } & \multicolumn{2}{|c|}{$\begin{array}{l}\text { Allele frequencies of } \\
\text { controls, } n,(\%)\end{array}$} \\
\hline & & & & & & & & $\mathrm{CC}$ & $\mathrm{CT}$ & TT & $\mathrm{C}$ & $\mathrm{T}$ & $\mathrm{CC}$ & $\mathrm{CT}$ & TT & C & $\mathrm{T}$ \\
\hline Konac [26] & 2007 & Caucasian & Turkey & PCR-RFLP & PB & 0.156 & $47 / 106$ & 1 & 13 & 33 & $15(16.0)$ & $79(84.0)$ & 1 & 34 & 71 & $36(17.0)$ & $176(83.0)$ \\
\hline $\begin{array}{l}\text { Jakubowska } \\
{[27]}\end{array}$ & 2008 & Caucasian & Poland & PCR-RFLP & $\mathrm{HB}$ & 0.863 & $145 / 280$ & 108 & 33 & 4 & $249(85.9)$ & $41(14.1)$ & 196 & 77 & 7 & $469(83.8)$ & $91(16.2)$ \\
\hline Jia [28] & 2009 & Asian & China & PCR-RFLP & $\mathrm{HB}$ & 0.729 & $256 / 329$ & 174 & 77 & 5 & $425(83.0)$ & $87(17.0)$ & 229 & 92 & 8 & $550(83.6)$ & $108(16.4)$ \\
\hline $\operatorname{Li}[29]$ & 2010 & Asian & China & PCR-RFLP & $\mathrm{HB}$ & 0.443 & $303 / 303$ & 211 & 86 & 6 & $508(83.8)$ & $98(16.2)$ & 201 & 94 & 8 & $496(81.8)$ & $110(18.2)$ \\
\hline $\begin{array}{l}\text { RinckJunior } \\
{[30]}\end{array}$ & 2015 & Caucasian & Brazil & PCR-RFLP & HB & 0.343 & $131 / 137$ & 111 & 16 & 4 & $238(90.8)$ & 24 (91.6) & 103 & 33 & 1 & $239(87.2)$ & $35(12.8)$ \\
\hline $\begin{array}{l}\text { Janardhan } \\
{[31]}\end{array}$ & 2015 & Asian & India & PCR-RFLP & $\mathrm{PB}$ & 0.625 & $300 / 320$ & 232 & 64 & 4 & $528(88.0)$ & $72(12.0)$ & 303 & 17 & 0 & $623(97.3)$ & $17(2.7)$ \\
\hline Zhang[32] & 2016 & Asian & China & PCR-RFLP & $\mathrm{HB}$ & 0.616 & $163 / 276$ & 109 & 46 & 8 & $264(81.0)$ & $62(19.0)$ & 192 & 75 & 9 & $459(83.1)$ & $93(16.9)$ \\
\hline \multicolumn{2}{|c|}{$-460 \mathrm{C} / \mathrm{T}(\mathrm{rs} 833061)$} & & & & & & & $\mathrm{CC}$ & CT & $\mathrm{TT}$ & C & $\mathrm{T}$ & $\mathrm{CC}$ & $\mathrm{CT}$ & TT & C & $\mathrm{T}$ \\
\hline Konac [26] & 2007 & Caucasian & Turkey & PCR-RFLP & PB & 0.156 & $47 / 106$ & 5 & 21 & 21 & $15(16.0)$ & $79(84.0)$ & 13 & 58 & 35 & 84 (39.6) & $128(60.4)$ \\
\hline Li [29] & 2010 & Asian & China & PCR-RFLP & HB & 0.262 & $303 / 303$ & 12 & 93 & 198 & $117(19.3)$ & $489(80.7)$ & 17 & 95 & 191 & $129(21.3)$ & 477 (78.7) \\
\hline $\begin{array}{l}\text { Janardhan } \\
\text { [31] }\end{array}$ & 2015 & Asian & India & PCR-RFLP & PB & 0.945 & $300 / 320$ & 96 & 152 & 52 & $344(57.3)$ & $256(42.7)$ & 167 & 128 & 25 & $462(72.2)$ & $178(27.8)$ \\
\hline Zhang[32] & 2016 & Asian & China & PCR-RFLP & $\mathrm{HB}$ & 0.002 & $163 / 176$ & 13 & 63 & 87 & $89(27.3)$ & $237(72.7)$ & 19 & 104 & 53 & $142(40.3)$ & $210(59.7)$ \\
\hline \multicolumn{2}{|c|}{$-2578 \mathrm{C} / \mathrm{A}(\mathrm{rs} 699947)$} & & & & & & & $\mathrm{CC}$ & $\mathrm{CA}$ & $\mathrm{AA}$ & $\mathrm{C}$ & A & $\mathrm{CC}$ & $\mathrm{CA}$ & AA & $\mathrm{C}$ & $\mathrm{A}$ \\
\hline Jia [28] & 2009 & Asian & China & PCR-RFLP & $\mathrm{HB}$ & 0.155 & $256 / 329$ & 140 & 99 & 17 & $379(74.0)$ & $133(26.0)$ & 191 & 113 & 25 & $495(75.2)$ & $163(24.8)$ \\
\hline $\operatorname{Li}[29]$ & 2010 & Asian & China & PCR-RFLP & HB & 0.807 & $303 / 303$ & 166 & 117 & 20 & $449(74.1)$ & $157(25.9)$ & 183 & 104 & 16 & 470 (77.6) & $136(22.4)$ \\
\hline $\begin{array}{l}\text { Janardhan } \\
{[31]}\end{array}$ & 2015 & Asian & India & PCR-RFLP & PB & 0.886 & $300 / 320$ & 116 & 142 & 42 & $374(62.3)$ & $226(37.7)$ & 117 & 154 & 49 & $388(60.6)$ & $252(39.4)$ \\
\hline Zhang[32] & 2016 & Asian & China & PCR-RFLP & $\mathrm{HB}$ & 0.257 & $163 / 276$ & 90 & 58 & 15 & $238(73.0)$ & $88(27.0)$ & 200 & 67 & 9 & $467(84.6)$ & $85(15.4)$ \\
\hline$-1154 \mathrm{G} / \mathrm{A}(\mathrm{rs} 15$ & 70360) & & & & & & & GG & GA & $\mathrm{AA}$ & $\mathrm{G}$ & A & GG & GA & $\mathrm{AA}$ & G & A \\
\hline $\operatorname{Li}[29]$ & 2010 & Asian & China & PCR-RFLP & $\mathrm{HB}$ & 0.952 & $302 / 303$ & 244 & 54 & 4 & $542(89.7)$ & $62(10.3)$ & 217 & 79 & 7 & $513(84.7)$ & $93(15.3)$ \\
\hline $\begin{array}{l}\text { Janardhan } \\
{[31]}\end{array}$ & 2015 & Asian & India & PCR-RFLP & PB & 0.425 & $300 / 320$ & 166 & 113 & 21 & $445(74.2)$ & $155(25.8)$ & 239 & 77 & 4 & $555(86.7)$ & $85(13.3)$ \\
\hline
\end{tabular}

PCR, polymerase chain reaction; RFLP, restriction fragment length polymorphism; HWE, Hardy-Weinberg equilibrium; PB, population-based; HB, hospital-based.

Table 2: Methodological quality of case-control studies in our meta-analyses, based on the Newcastle-Ottawa Scale

\begin{tabular}{|c|c|c|c|c|c|c|c|c|c|}
\hline \multirow[b]{2}{*}{ Study } & \multicolumn{4}{|c|}{ Selection (score) } & \multirow{2}{*}{$\begin{array}{c}\text { Comparability } \\
\text { (score) }\end{array}$} & \multicolumn{3}{|c|}{ Exposure (score) } & \multirow[b]{2}{*}{ Total score } \\
\hline & $\begin{array}{c}\text { Adequate } \\
\text { definition of } \\
\text { patient cases }\end{array}$ & $\begin{array}{c}\text { Representativeness of } \\
\text { patients/cases }\end{array}$ & $\begin{array}{l}\text { Selection of } \\
\text { controls }\end{array}$ & $\begin{array}{l}\text { Definition of } \\
\text { controls }\end{array}$ & & $\begin{array}{c}\text { Ascertainment } \\
\text { of exposure } \\
\text { (blinding) }\end{array}$ & $\begin{array}{l}\text { Same method of } \\
\text { ascertainment for } \\
\text { participants }\end{array}$ & $\begin{array}{l}\text { Non- } \\
\text { response } \\
\text { rate }^{\mathrm{a}}\end{array}$ & \\
\hline Konac [26] & 1 & 1 & 1 & 1 & 2 & 0 & 1 & 1 & 8 \\
\hline Jakubowska [27] & 1 & 1 & 0 & 1 & 2 & 0 & 1 & 1 & 7 \\
\hline Jia [28] & 1 & 1 & 0 & 1 & 1 & 0 & 1 & 1 & 6 \\
\hline $\operatorname{Li}[29]$ & 1 & 1 & 0 & 1 & 1 & 0 & 1 & 1 & 6 \\
\hline Rinck-Junior [30] & 1 & 1 & 0 & 1 & 1 & 0 & 1 & 1 & 6 \\
\hline Janardhan [31] & 1 & 1 & 1 & 1 & 2 & 0 & 1 & 1 & 8 \\
\hline Zhang [32] & 1 & 1 & 1 & 1 & 2 & 0 & 1 & 1 & 8 \\
\hline
\end{tabular}

${ }^{a}$ One point was awarded when there was no significant difference in the response rate between groups, based on the chi-squared test $(P>0.05)$.

${ }^{\mathrm{b}}$ Calculated by adding up the points awarded for each item.

(Figure 2D); and heterozygous model, OR 1.07, 95\% CI $0.65-1.76, P=0.79$ (Figure 2E).

We also meta-analyzed data for ethnic subgroups. Meta-analysis of 4 studies [28, 29, 31, 32] involving 1,022 Asian cases and 1,228 Asian controls showed no evidence of a significant association between the
$+936 \mathrm{C} / \mathrm{T}$ polymorphism and ovarian risk risk for any of the five genetic models (Table 3): allelic, OR 1.47, 95\% CI $0.81-2.67, P=0.21$; recessive model, OR $1.19,95 \%$ CI $0.68-2.11, P=0.54$; dominant, OR $0.67,95 \%$ CI 0.35 $1.29, P=0.23$; homozygous, OR $1.22,95 \%$ CI 0.69-2.16, $P=0.50$; and heterozygous, OR $1.46,95 \%$ CI $0.77-$ 
Table 3: Overall meta-analysis of the association between the $+936 \mathrm{C} / \mathrm{T}$ (rs3025039) and risk of ovarian cancer

\begin{tabular}{|c|c|c|c|c|c|c|}
\hline \multirow{2}{*}{$\begin{array}{l}\text { Genotype comparison and } \\
\text { genetic model }\end{array}$} & \multirow{2}{*}{ OR $[95 \% \mathrm{CI}]$} & \multirow{2}{*}{$\mathrm{Z}$ (P value) } & \multicolumn{3}{|c|}{$\begin{array}{c}\text { Heterogeneity of study } \\
\text { design }\end{array}$} & \multirow{2}{*}{$\begin{array}{c}\text { Analysis } \\
\text { model }\end{array}$} \\
\hline & & & $\chi^{2}$ & $\begin{array}{c}\text { df }(P \\
\text { value })\end{array}$ & $\begin{array}{l}I^{2} \\
(\%)\end{array}$ & \\
\hline \multicolumn{7}{|c|}{$+936 \mathrm{C} / \mathrm{T}$ (rs3025039) in total population from 7 case control studies ( 1,345 cases and 1,751 controls) } \\
\hline Allelic (T-allele vs. C-allele) & $1.17[0.79,1.72]$ & $0.78(0.44)$ & 37.23 & $6(<0.001)$ & 84 & Random \\
\hline Recessive (TT vs. CT + CC) & $1.25[0.82,1.88]$ & $1.04(0.30)$ & 4.82 & $6(0.57)$ & 0 & Fixed \\
\hline Dominant $(\mathrm{CC}$ vs. $\mathrm{CT}+\mathrm{TT})$ & $0.89[0.55,1.45]$ & $0.45(0.65)$ & 39.45 & $6(<0.001)$ & 85 & Random \\
\hline Homozygous (TT vs. CC) & $1.24[0.76,2.03]$ & $0.87(0.39)$ & 5.50 & $6(0.48)$ & 0 & Fixed \\
\hline Heterozygous (CT vs. CC) & $1.07[0.65,1.76]$ & $0.27(0.79)$ & 38.83 & $6(<0.001)$ & 85 & Random \\
\hline \multicolumn{7}{|c|}{ +936C/T (rs3025039) in Asian population from 4 case-control studies (1,022 cases and 1,228 controls) } \\
\hline Allelic (T-allele vs. C-allele) & $1.47[0.81,2.67]$ & $1.25(0.21)$ & 33.47 & $3(<0.001)$ & 91 & Random \\
\hline Recessive (TT vs. CT $+\mathrm{CC}$ ) & $1.19[0.68,2.11]$ & $0.61(0.54)$ & 3.46 & $3(0.33)$ & 13 & Fixed \\
\hline Dominant (CC vs. CT + TT) & $0.67[0.35,1.29]$ & $1.19(0.23)$ & 30.92 & $3(<0.001)$ & 90 & Random \\
\hline Homozygous (TT vs. CC) & $1.22[0.69,2.16]$ & $0.68(0.50)$ & 3.96 & $3(0.27)$ & 24 & Fixed \\
\hline Heterozygous (CT vs. CC) & $1.46[0.77,2.75]$ & $1.16(0.24)$ & 27.99 & $3(<0.001)$ & 89 & Random \\
\hline \multicolumn{7}{|c|}{$+936 \mathrm{C} / \mathrm{T}$ (rs3025039) in Caucasian population from 3 case-control studies (323 cases and 523 controls) } \\
\hline Allelic (T-allele vs. C-allele) & $0.84[0.63,1.12]$ & $1.19(0.23)$ & 1.05 & $2(0.59)$ & 0 & Fixed \\
\hline Recessive (TT vs. CT + CC) & $1.31[0.71,2.39]$ & $0.87(0.38)$ & 1.28 & $2(0.53)$ & 0 & Fixed \\
\hline Dominant (CC vs. CT + TT) & $1.44[1.00,2.07]$ & $1.98(0.05)$ & 1.07 & $2(0.59)$ & 0 & Fixed \\
\hline Homozygous (TT vs. CC) & $1.31[0.50,3.44]$ & $0.55(0.58)$ & 1.51 & $2(0.47)$ & 0 & Fixed \\
\hline Heterozygous (CT vs. CC) & $0.64[0.44,0.93]$ & $2.33(0.02)$ & 1.90 & $2(0.39)$ & 0 & Fixed \\
\hline \multicolumn{7}{|c|}{$+936 \mathrm{C} / \mathrm{T}$ (rs3025039) in Chinese population from 3 case-control studies ( 722 cases and 908 controls) } \\
\hline Allelic (T-allele vs. C-allele) & $1.00[0.83,1.20]$ & $0.01(0.99)$ & 1.57 & $2(0.46)$ & 0 & Fixed \\
\hline Recessive (TT vs. CT + CC) & $1.00[0.55,1.83]$ & $0.00(1.00)$ & 1.18 & $2(0.55)$ & 0 & Fixed \\
\hline Dominant (CC vs. $\mathrm{CT}+\mathrm{TT})$ & $1.00[0.81,1.23]$ & $0.01(0.99)$ & 1.28 & $2(0.53)$ & 0 & Fixed \\
\hline Homozygous (TT vs. CC) & $1.00[0.55,1.84]$ & $0.01(1.00)$ & 1.29 & $2(0.52)$ & 0 & Fixed \\
\hline Heterozygous (CT vs. CC) & $1.00[0.81,1.24]$ & $0.01(0.99)$ & 0.99 & $2(0.61)$ & 0 & Fixed \\
\hline
\end{tabular}

OR, odds ratio; $95 \%$ CI, $95 \%$ confidence interval.

$2.75, P=0.24$. Similarly, no evidence of an association was identified in meta-analysis of 3 studies [26, 27, 30] involving 323 Caucasian cases and 523 Caucasian controls in four genetic models: allelic, OR $0.84,95 \%$ CI $0.63-$
$1.12, P=0.23$; recessive, OR $1.31,95 \%$ CI $0.71-2.39$, $P=0.38$; dominant, OR $1.44,95 \%$ CI $1.00-2.07, P=0.05$; and homozygous, OR $1.31,95 \%$ CI $0.50-3.44, P=0.58$. In contrast, the $\mathrm{CT}$ genotype at $+936 \mathrm{C} / \mathrm{T}$ was found to be a 


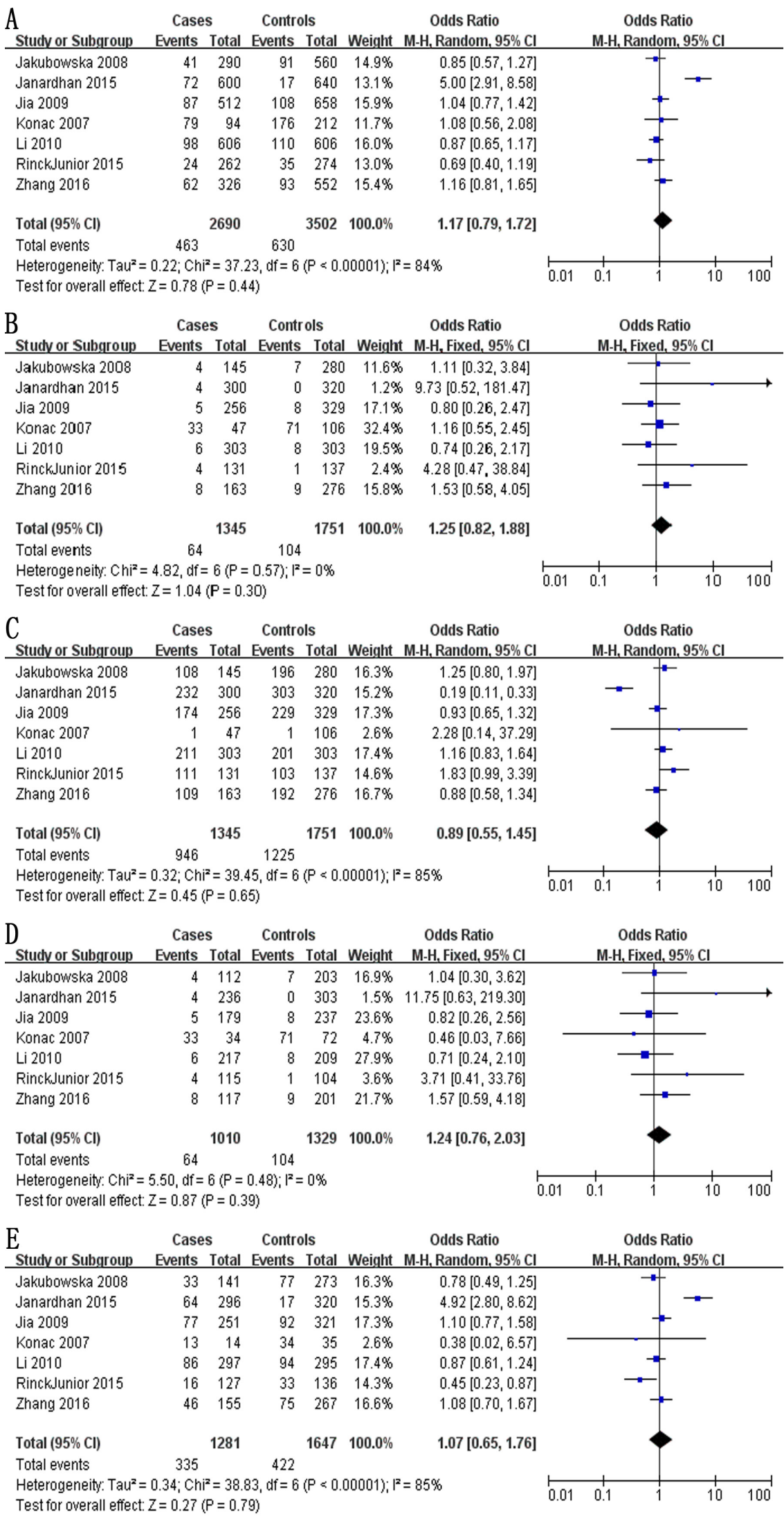

Figure 2: Forest plot describing the association between the $+936 \mathrm{C} / \mathrm{T}$ polymorphism ( $\mathrm{rs3025039})$ and risk of ovarian cancer across all study participants according to five genetic models. (A) allelic (T-allele vs. C-allele), (B) recessive (TT vs. $\mathrm{CT}+\mathrm{CC}),(\mathbf{C})$ dominant $(\mathrm{CC}$ vs. CT + TT), (D) homozygous (TT vs. CC) and (E) heterozygous (CT vs. CC). 
Table 4: Overall meta-analysis of the association between the $-460 \mathrm{C} / \mathrm{T}$ (rs833061) and risk of ovarian cancer

\begin{tabular}{|c|c|c|c|c|c|c|}
\hline \multirow{2}{*}{$\begin{array}{c}\text { Genotype comparison and } \\
\text { genetic model }\end{array}$} & \multirow{2}{*}{ OR $[95 \% \mathrm{CI}]$} & \multirow{2}{*}{$\mathrm{Z}$ (P value) } & \multicolumn{3}{|c|}{ Heterogeneity of study design } & \multirow{2}{*}{$\begin{array}{c}\text { Analysis } \\
\text { model }\end{array}$} \\
\hline & & & $\chi^{2}$ & df $(P$ value $)$ & $I^{2}(\%)$ & \\
\hline \multicolumn{7}{|c|}{$-460 \mathrm{C} / \mathrm{T}$ (rs833061) in total population from 4 case-control studies ( 813 cases and 905 controls) } \\
\hline Allelic (T-allele vs. C-allele) & $1.80[1.26,2.59]$ & $3.20(0.001)$ & 14.46 & $3(0.002)$ & 79 & Random \\
\hline Recessive (TT vs. CT + CC) & $1.84[1.13,2.98]$ & $2.47(0.01)$ & 12.41 & $3(0.006)$ & 76 & Random \\
\hline Dominant (CC vs. CT + TT) & $0.51[0.39,0.67]$ & $4.87(<0.001)$ & 3.30 & $3(0.35)$ & 9 & Fixed \\
\hline Homozygous (TT vs. CC) & $2.48[1.72,3.56]$ & $4.90(<0.001)$ & 4.30 & $3(0.23)$ & 30 & Fixed \\
\hline Heterozygous (CT vs. CC) & $1.67[1.26,2.21]$ & $3.35(<0.001)$ & 5.24 & $3(0.16)$ & 43 & Fixed \\
\hline \multicolumn{7}{|c|}{$-460 \mathrm{C} / \mathrm{T}$ (rs833061) in Asian population from 3 case-control studies ( 766 cases and 799 controls) } \\
\hline Allelic (T-allele vs. C-allele) & $1.58[1.13,2.22]$ & $2.65(0.008)$ & 8.85 & $2(0.01)$ & 77 & Random \\
\hline Recessive (TT vs. CT + CC) & $1.90[1.03,3.49]$ & $2.06(0.04)$ & 12.40 & $2(0.002)$ & 84 & Random \\
\hline Dominant (CC vs. $\mathrm{CT}+\mathrm{TT})$ & $0.49[0.37,0.65]$ & $4.97(<0.001)$ & 2.41 & $2(0.30)$ & 17 & Fixed \\
\hline Homozygous (TT vs. CC) & $2.61[1.78,3.82]$ & $4.93(<0.001)$ & 3.62 & $2(0.16)$ & 45 & Fixed \\
\hline Heterozygous (CT vs. CC) & $1.73[1.29,2.32]$ & $3.69(<0.001)$ & 4.21 & $2(0.12)$ & 53 & Fixed \\
\hline
\end{tabular}

OR, odds ratio; $95 \% \mathrm{CI}, 95 \%$ confidence interval.

protective factor in the heterozygous model (OR $0.64,95 \%$ CI $0.44-0.93, P=0.02$; Table 3). Lastly, meta-analysis of 3 studies $[28,29,32]$ involving 722 Chinese cases and 908 Chinese controls showed no evidence of a significant association between the $+936 \mathrm{C} / \mathrm{T}$ polymorphism and ovarian risk for any of the five genetic models (Table 3): allelic, OR 1.00, 95\% CI 0.83-1.20, $P=099$; recessive, OR $1.00,95 \%$ CI $0.55-1.83, P=1.00$; dominant, OR 1.00 , $95 \%$ CI $0.81-1.23, P=0.99$; homozygous, OR $1.00,95 \%$ CI $0.55-1.84, P=1.00$; and heterozygous, OR $1.00,95 \%$ CI $0.81-1.24, P=0.99$.

\section{Meta-analysis of studies on the $-460 \mathrm{C} / \mathrm{T}$ (rs833061) polymorphism}

The meta-analysis of a possible association between the $-460 \mathrm{C} / \mathrm{T}$ polymorphism and ovarian risk is summarized in Table 4. Based on the total study population involving 813 cases and 905 controls, a significant association between the $-460 \mathrm{C} / \mathrm{T}$ polymorphism and ovarian risk was demonstrated across the total population according to five genetic models: allelic, OR $1.80,95 \%$ CI 1.26 $2.59, P=0.001$ (Figure 3A); recessive, OR 1.84, 95\% CI 1.13-2.98, $P=0.01$ (Figure 3B); dominant, OR 0.51, 95\% CI 0.39-0.67, $P<0.001$ (Figure 3C); homozygous, OR 2.48, 95\% CI 1.72-3.56, $P<0.001$ (Figure 3D); and heterozygous, OR $1.67,95 \%$ CI $1.26-2.21, P<0.001$ (Figure 3E).

A significant association was also observed in the subgroup of 766 Asian cases and 799 Asian controls in 3 studies [29-32] according to five genetic models (Table 4): allelic, OR 1.58, 95\% CI 1.13-2.22, $P=0.008$; recessive, OR 1.90, 95\% CI 1.03-3.49, $P=0.04$; dominant, OR 0.49 , 95\% CI 0.37-0.65, $P<0.001$; homozygous, OR $2.61,95 \%$ CI 1.78-3.82, $P<0.001$; and heterozygous, OR $1.73,95 \%$ CI 1.29-2.32, $P<0.001$.

\section{Meta-analysis of studies on the -2578C/A (rs699947) polymorphism}

The meta-analysis of a possible association between the $-2578 \mathrm{C} / \mathrm{A}$ polymorphism and ovarian cancer risk is summarized in Table 5. Based on the total study population (exclusively Asian) involving 1,022 cases and 1,228 controls, no evidence of an association was identified in four genetic models: allelic, OR 1.23, 95\% CI 0.91-1.66, $P=0.18$ (Figure 4A); recessive, OR $1.11,95 \%$ CI $0.83-$ $1.50, P=0.48$ (Figure 4B); dominant, OR $0.78,95 \% \mathrm{CI}$ $0.57-1.08, P=0.14$ (Figure 4C); and homozygous, OR $1.33,95 \% \mathrm{CI} 0.75-2.35, P=0.33$ (Figure 4D). In contrast, the CA genotype at $-2578 \mathrm{C} / \mathrm{A}$ was found to be a risk factor in the heterozygous model (OR 1.22, 95\% CI 1.02-1.46, $P=0.03$; Figure 4E).

\section{Meta-analysis of studies on the -1154G/A (rs1570360) polymorphism}

The meta-analysis of a possible association between the $-1154 \mathrm{G} / \mathrm{A}$ polymorphism and ovarian cancer risk is summarized in Table 6 . Based on the total study population (exclusively Asian) involving 602 cases and 623 controls, none of the five genetic models indicated a significant 
A

Cases Controls Odds Ratio Odds Ratio

Study or Subgroup Events Total Events Total Weight M-H, Random, 95\% Cl

$1.93[1.52,2.45]$

$\begin{array}{lrrrrrr}\text { Janardhan 2015 } & 256 & 600 & 178 & 640 & 29.1 \% & 1.93[1.52,2.45] \\ \text { Konac 2007 } & 79 & 94 & 128 & 212 & 16.9 \% & 3.46[1.87,6.40]\end{array}$

$\begin{array}{lllllll}\text { Li } 2010 & 489 & 606 & 477 & 606 & 27.7 \% & 1.13[0.85,1.50]\end{array}$

Zhang 2010

$\begin{array}{lllll}489 & 606 & 477 & 606 & 27.7 \%\end{array}$

$1.80[1.30,2.49]$

$\begin{array}{lllll}\text { Total }(95 \% \mathrm{Cl}) \quad 1626 & 1810 & 100.0 \% & 1.80[1.26,2.59]\end{array}$

$\begin{array}{lcc}\text { Total events } \quad 1061 & 993 \\ \text { Heterogeneity: } \text { Ta }^{2}=0.10 ; \mathrm{Chi}^{2}=14.46, \mathrm{df}=3(P=0.002) ; 1^{2}=79 \%\end{array}$

Test for overall effect: $Z=3.20(P=0.001)$

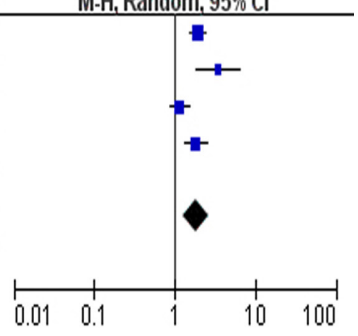

B

\begin{tabular}{|c|c|c|c|c|c|c|}
\hline Studv or Subgroup & Events & Total & Events & & Weight & M-H, Random, $95 \% \mathrm{Cl}$ \\
\hline Janardhan 2015 & 52 & 300 & 25 & 320 & $24.7 \%$ & $2.47[1.49,4.10]$ \\
\hline Konac 2007 & 21 & 47 & 35 & 106 & $19.7 \%$ & $1.64[0.81,3.31]$ \\
\hline Li 2010 & 198 & 303 & 191 & 303 & $29.2 \%$ & $1.11[0.79,1.54]$ \\
\hline Zhang 2016 & 87 & 163 & 53 & 176 & $26.3 \%$ & $2.66[1.70,4.15]$ \\
\hline Total (95\% Cl) & & 813 & & 905 & $100.0 \%$ & $1.84[1.13,2.98]$ \\
\hline Total events & 358 & & 304 & & & \\
\hline \multicolumn{7}{|c|}{ Heterogeneity: Tau $^{2}=0.18 ; \mathrm{Chi}^{2}=12.41, \mathrm{df}=3(\mathrm{P}=0.006)_{1} \mathrm{I}^{2}=76 \%$} \\
\hline
\end{tabular}

Odds Ratio

M.H, Random, $95 \% \mathrm{Cl}$

C Studv or Subgroup Events Total Events Total Weight M-H, Fixed, 95\% Cl $\begin{array}{lllllll}\text { Janardhan } 2015 & 96 & 300 & 167 & 320 & 73.2 \% & 0.43[0.31,0.60]\end{array}$ Konac $200 ?$

Li 2010

Zhang 2016

$\begin{array}{llllll}5 & 47 & 13 & 106 & 4.8 \% & 0.85[0.29,2.54]\end{array}$

$\begin{array}{lllllll}12 & 303 & 17 & 303 & 10.9 \% & 0.69[0.33,1.48]\end{array}$

$\begin{array}{lllllll}13 & 163 & 19 & 176 & 11.2 \% & 0.72[0.34,1.50]\end{array}$

$\begin{array}{lllllll}\text { Total }(95 \% \mathrm{Cl}) & & 813 & 905 & 100.0 \% & 0.51[0.39,0.67] \\ \text { Total events } & 126 & & 216 & & \end{array}$

Heterogeneity. $C \mathrm{Ch}^{2}=3.30, \mathrm{df}=3(\mathrm{P}=0.35) \mathrm{I}^{2}=9 \%$

Test for overall effect: $Z=4.87$ ( $P<0.00001)$

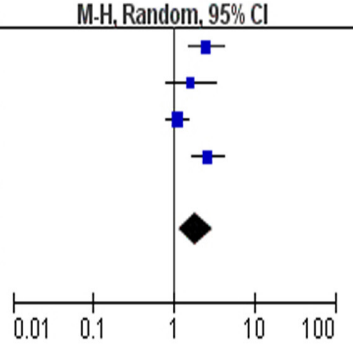

D Cases Controls Odds Ratio Odds Ratio

Studv or Subgroup Events Total Events Total Weight M-H, Fixed, 95\% Cl M-H, Fixed, 95\% Cl

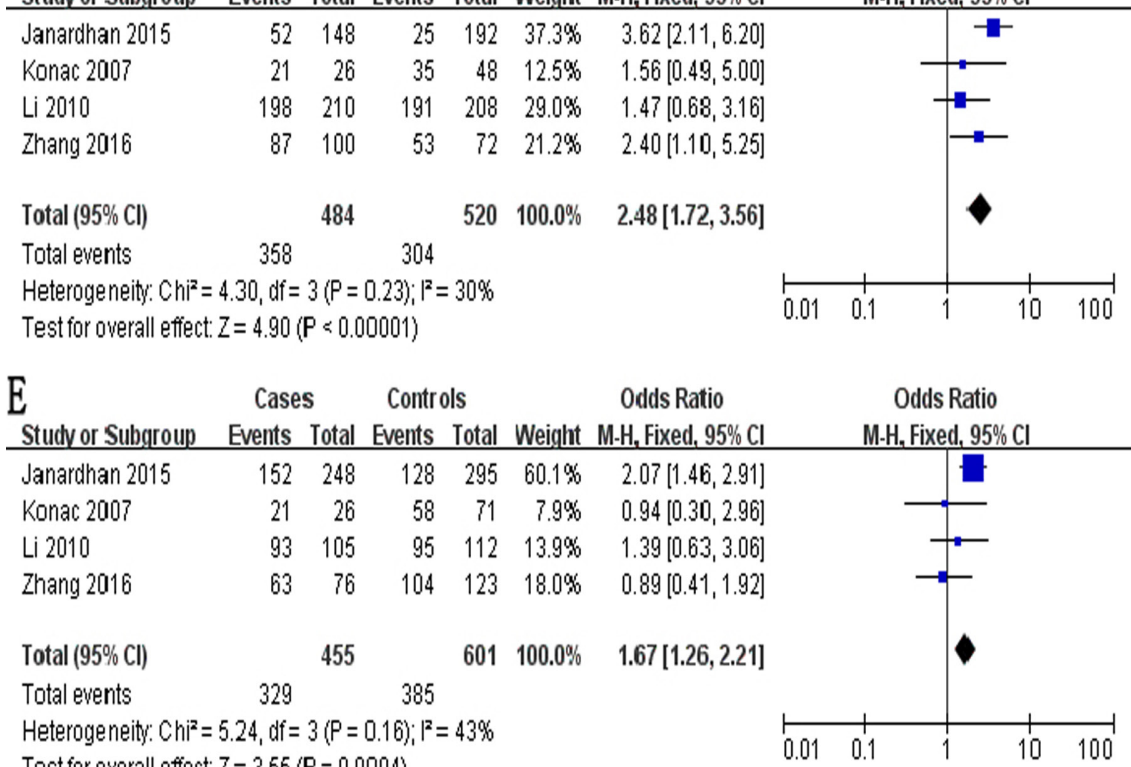

Figure 3: Forest plot describing the association between the $-460 \mathrm{C} / \mathrm{T}$ polymorphism (rs833061) and risk of ovarian cancer across all study participants according to five genetic models. (A) allelic (T-allele vs. C-allele), (B) recessive (TT vs. CT + CC), (C) dominant (CC vs. CT + TT), (D) homozygous (TT vs. CC) and (E) heterozygous (CT vs. CC). 
Table 5: Overall meta-analysis of the association between the -2578C/A (rs699947) and risk of ovarian cancer

\begin{tabular}{|c|c|c|c|c|c|c|}
\hline \multirow{2}{*}{$\begin{array}{c}\text { Genotype comparison and } \\
\text { genetic model }\end{array}$} & \multirow{2}{*}{ OR $[95 \% \mathrm{CI}]$} & \multirow{2}{*}{$\mathrm{Z}$ (P value) } & \multicolumn{3}{|c|}{$\begin{array}{c}\text { Heterogeneity of study } \\
\text { design }\end{array}$} & \multirow{2}{*}{$\begin{array}{c}\text { Analysis } \\
\text { model }\end{array}$} \\
\hline & & & $\chi^{2}$ & $\begin{array}{c}\operatorname{df}(P \\
\text { value })\end{array}$ & $\mathbf{I}^{2}(\%)$ & \\
\hline \multicolumn{7}{|c|}{$-2578 \mathrm{C} / \mathrm{A}$ (rs699947) in total population from 4 case-control studies (1,022 cases and 1,228 controls) } \\
\hline Allelic (A-allele vs. C-allele) & $1.23[0.91,1.66]$ & $1.34(0.18)$ & 14.70 & $3(0.002)$ & 80 & Random \\
\hline Recessive (AA vs. CA + CC) & $1.11[0.83,1.50]$ & $0.71(0.48)$ & 6.85 & $3(0.08)$ & 56 & Fixed \\
\hline Dominant $(\mathrm{CC}$ vs. $\mathrm{CA}+\mathrm{AA})$ & $0.78[0.57,1.08]$ & $1.48(0.14)$ & 10.46 & $3(0.02)$ & 71 & Random \\
\hline Homozygous (AA vs. CC) & $1.33[0.75,2.35]$ & $0.97(0.33)$ & 9.04 & $3(0.03)$ & 67 & Random \\
\hline Heterozygous (CA vs. CC) & $1.22[1.02,1.46]$ & $2.20(0.03)$ & 6.72 & $3(0.08)$ & 55 & Fixed \\
\hline
\end{tabular}

OR, odds ratio; $95 \%$ CI, 95\% confidence interval.

association: allelic, OR $1.20,95 \%$ CI $0.34-4.22, P=0.77$ (Figure 5A); recessive, OR 1.87, 95\% CI 0.19-18.93, $P=0.59$ (Figure 5B); dominant, OR 0.83, 95\% CI 0.22 $3.22, P=0.79$ (Figure 5C); homozygous, OR $1.99,95 \%$ CI 0.14-28.35, $P=0.61$ (Figure 5D); and heterozygous, OR $1.14,95 \%$ CI $0.34-3.85, P=0.84$ (Figure 5E).

\section{Sensitivity analysis}

The robustness of the meta-analysis of 4 studies $[26,29,31,32]$ examining a possible association between the $-460 \mathrm{C} / \mathrm{T}$ polymorphism and ovarian cancer risk was assessed by repeating the meta-analysis after excluding a study by Zhang et al. [32] in which the $P$ value associated with HWE was less than 0.05 . Deleting these data from the meta-analysis did not alter the results except in the recessive model, the results of which should therefore be interpreted with caution.

\section{Publication bias}

Potential publication bias in this meta-analysis was assessed using Begg's funnel plot. No obvious asymmetry was observed in Begg's funnel plots of allelic modeling of the polymorphisms $+936 \mathrm{C} / \mathrm{T}$ (Figure 6), $-460 \mathrm{C} / \mathrm{T}$ (Figure 7) or -2578C/A (Figure 8). $P$ values for Begg's test were greater than 0.05 for the $+936 \mathrm{C} / \mathrm{T}$ results based on all the genetic models: allelic, $P=0.230$; recessive, $P=0.230$; dominant, $P=1.000$; homozygous, $P=$ 0.368 ; and heterozygous, $P=0.764$. Similarly, $P$ values were greater than 0.05 for the $-460 \mathrm{C} / \mathrm{T}$ results (allelic, $P=0.734$; recessive, $P=1.000$; dominant, $P=0.734$; homozygous, $P=0.734$; heterozygous, $P=0.734$ ) and for the $-2578 \mathrm{C} / \mathrm{A}$ results (allelic, $P=0.308$; recessive, $P=0.089$; dominant, $P=0.734$; homozygous, $P=0.089$; heterozygous, $P=0.734$ ). These results suggest no potential publication bias in the included data on $+936 \mathrm{C} / \mathrm{T}$, $-460 \mathrm{C} / \mathrm{T}$ and $-2578 \mathrm{C} / \mathrm{A}$ polymorphisms. Begg's test was not applied to data on the $-1154 \mathrm{G} / \mathrm{A}$ polymorphism because of the small number of publications.

\section{DISCUSSION}

The number of case-control studies exploring the influence of VEGF-A polymorphisms on ovarian cancer risk has grown in recent years [26-32]. Limited sample size and ethnic differences among the various populations examined have contributed to a lack of consensus in this literature, so we conducted this comprehensive metaanalysis to evaluate the association of ovarian cancer risk with four polymorphisms in the VEGF-A gene $(+936 \mathrm{C} / \mathrm{T}$, $-460 \mathrm{C} / \mathrm{T},-2578 \mathrm{C} / \mathrm{A}$ and $-1154 \mathrm{G} / \mathrm{A})$. Our meta-analysis suggests that the $-460 \mathrm{C} / \mathrm{T}$ polymorphism is significantly associated with ovarian cancer risk across the total population as well as the Asian population. In contrast, none of the five genetic models suggested a significant association between the $+936 \mathrm{C} / \mathrm{T}$ polymorphism and ovarian cancer risk in Asian populations in general or in Chinese populations specifically. None of the five genetic models suggested a significant association between the $-1154 \mathrm{G} / \mathrm{A}$ polymorphism and ovarian cancer risk across the entire study population.

While the present meta-analysis was being conducted, Zhang et al. [33] published a meta-analysis of the relationship between ovarian cancer risk and the three polymorphisms $+936 \mathrm{C} / \mathrm{T},-460 \mathrm{C} / \mathrm{T}$, and $-2578 \mathrm{C} / \mathrm{A}$. Similar to their results, we found that the CT genotype at $+936 \mathrm{C} / \mathrm{T}$ may act as a protective factor in Caucasian populations. On the other hand, our meta-analysis contrasts with the previous one because we found the $-460 \mathrm{C} / \mathrm{T}$ polymorphism to be significantly associated with ovarian cancer risk across the total population as well as the Asian subpopulation, and the CA genotype at $-2578 \mathrm{C} / \mathrm{A}$ to be associated with cancer risk across the total population, whereas that previous metaanalysis did not report either association. This discrepancy 


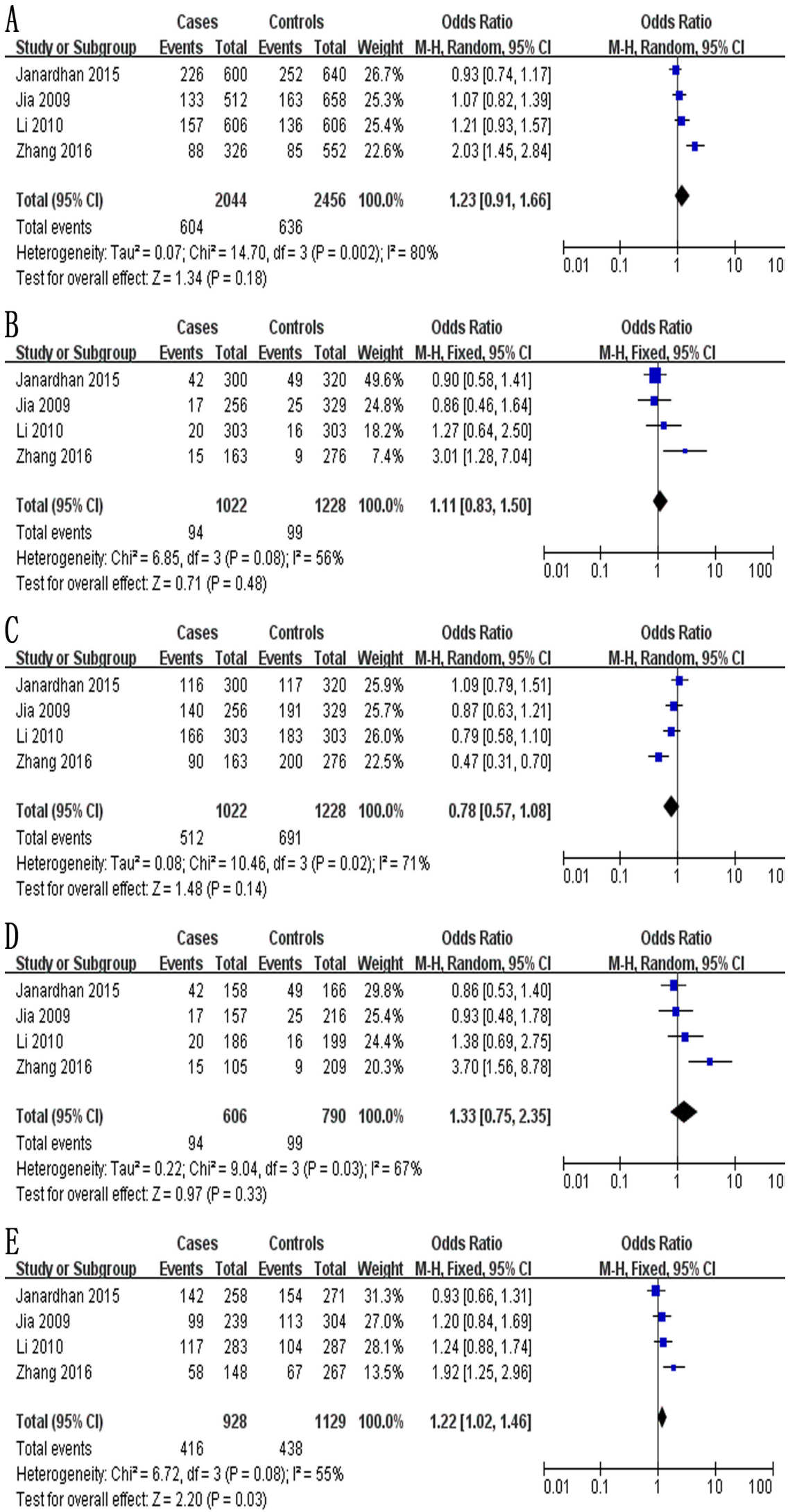

Figure 4: Forest plot describing the association between the -2578C/A polymorphism (rs699947) and risk of ovarian cancer across all study participants according to five genetic models. (A) allelic (A-allele vs. C-allele), (B) recessive (AA vs. CA + CC), (C) dominant (CC vs. CA + AA), (D) homozygous (AA vs. CC) and (E) heterozygous (CA vs. CC). 


\section{Table 6: Overall meta-analysis of the association between the -1154G/A (rs1570360) and risk of ovarian cancer}

\begin{tabular}{|c|c|c|c|c|c|c|}
\hline \multirow{2}{*}{$\begin{array}{l}\text { Genotype comparison and } \\
\text { genetic model }\end{array}$} & \multirow{2}{*}{ OR $[95 \% \mathrm{CI}]$} & \multirow{2}{*}{$\mathrm{Z}$ (P value) } & \multicolumn{3}{|c|}{ Heterogeneity of study design } & \multirow{2}{*}{ Analysis model } \\
\hline & & & $\chi^{2}$ & df (P value) & $I^{2}(\%)$ & \\
\hline \multicolumn{7}{|c|}{$-1154 \mathrm{G} / \mathrm{A}(\mathrm{rs} 1570360)$ in total population from 2 case-control studies (602 cases and 623 controls) } \\
\hline Allelic (A-allele vs. G-allele) & $1.20[0.34,4.22]$ & $0.29(0.77)$ & 31.05 & $1(<0.001)$ & 97 & Random \\
\hline Recessive (AA vs. GC + GG) & $1.87[0.19,18.93]$ & $0.53(0.59)$ & 7.91 & $1(0.005)$ & 87 & Random \\
\hline Dominant (GG vs. GA + AA) & $0.83[0.22,3.22]$ & $0.26(0.79)$ & 28.14 & $1(<0.001)$ & 96 & Random \\
\hline Homozygous (AA vs. GG) & $1.99[0.14,28.35]$ & $0.51(0.61)$ & 10.34 & $1(0.001)$ & 90 & Random \\
\hline Heterozygous (GA vs. GG) & $1.14[0.34,3.85]$ & $0.21(0.84)$ & 21.58 & $1(<0.001)$ & 95 & Random \\
\hline
\end{tabular}

OR, odds ratio; $95 \% \mathrm{CI}, 95 \%$ confidence interval.

may reflect the fact that we included two large case-control studies involving all four VEGF-A polymorphisms absent from the previous meta-analysis, leading to much larger sample sizes for meta-analysis of $2578 \mathrm{C} / \mathrm{A}$ and $-460 \mathrm{C} / \mathrm{T}$ polymorphisms in our work. In addition, we meta-analyzed the relationship between $-1154 \mathrm{G} / \mathrm{A}$ polymorphism and ovarian risk, which was not examined in that previous meta-analysis. Therefore, our meta-analysis provides new evidence for the important role of VEGF-A polymorphisms in ovarian cancer development. To the best of our knowledge, the present study is the most comprehensive and robust meta-analysis of these genetic polymorphisms and ovarian cancer.

Despite the potential insights it offers, the present study has several limitations that may affect interpretation of the results. First, the $P$ value for HWE was less than 0.05 in the case-control study by Zhang et al. [32] on the $-460 \mathrm{C} /$ $\mathrm{T}$ polymorphism. These results suggest that this study population may not be representative of the broader target population. Nevertheless, sensitivity analyses showed that deleting these data from the meta-analysis did not alter the results except in the recessive model, which is unlikely to significantly affect the observed significant relationship between $-460 \mathrm{C} / \mathrm{T}$ polymorphism and ovarian cancer risk. Second, our exclusion of unpublished data and of papers published in languages other than English and Chinese may have biased our results. Third, the studies may be subject to performance bias, attrition bias and reporting bias, although Newcastle-Ottawa scores were at least 6 for all 7 studies, indicating high quality. Lastly, the results may be affected by additional confounding factors, such as age, obesity, type of cancer, or other factors, and we could not take this into account in the meta-analyses because studies either did not report these baseline data or they aggregated the data in different ways. Thus, these conclusions should be verified in large, well-designed studies.

In conclusion, this meta-analysis indicates that there may be a significant association between the $-460 \mathrm{C} / \mathrm{T}$ polymorphism and ovarian cancer risk. The
CA genotype at $-2578 \mathrm{C} / \mathrm{A}$ may be a risk factor in the total population, while the $\mathrm{CT}$ genotype at $+936 \mathrm{C} / \mathrm{T}$ may be a protective factor in the Caucasian population. The $-1154 \mathrm{G} / \mathrm{A}$ polymorphism may not be related to ovarian cancer risk.

\section{MATERIALS AND METHODS}

\section{Search strategy}

PubMed, EMBASE, Google Scholar and the Chinese National Knowledge Infrastructure databases were systematically searched up to April 12, 2017 for clinical and experimental case-control studies published in English or Chinese that assessed potential associations of ovarian cancer risk with at least one of the following polymorphisms in the VEGF-A gene: $+936 \mathrm{C} / \mathrm{T}$ (rs3025039), $-460 \mathrm{C} / \mathrm{T}$ (rs833061), -2578C/A (rs699947), and $-1154 \mathrm{G} / \mathrm{A}$ (rs1570360). The following search strings were used: vascular endothelial growth factor $+936 \mathrm{C} / \mathrm{T}$, vascular endothelial growth factor $-460 \mathrm{C} / T$, vascular endothelial growth factor $-2578 \mathrm{C} / \mathrm{A}$, vascular endothelial growth factor -1154G/A, rs3025039, rs833061, rs699947, and $r s 1570360$. Searches were also conducted with each of these eight terms AND each of the following terms: polymorphism, polymorphisms, SNP, variant, variants, variation, genotype, genetic or mutation. Lastly, searches were conducted with each of the above terms AND each of the following: ovarian cancer, ovarian carcinoma or OC. Reference lists in identified articles and reviews were also searched manually to identify additional eligible studies.

This literature and meta-analysis were performed in accordance with the guidelines and recommendations of the Preferred Reporting Items for Systematic Reviews and Meta-Analyses (Supplementary Table 1) [34].

\section{Inclusion criteria}

To be included in our review and meta-analysis, studies had to satisfy the following criteria: (1) a case- 


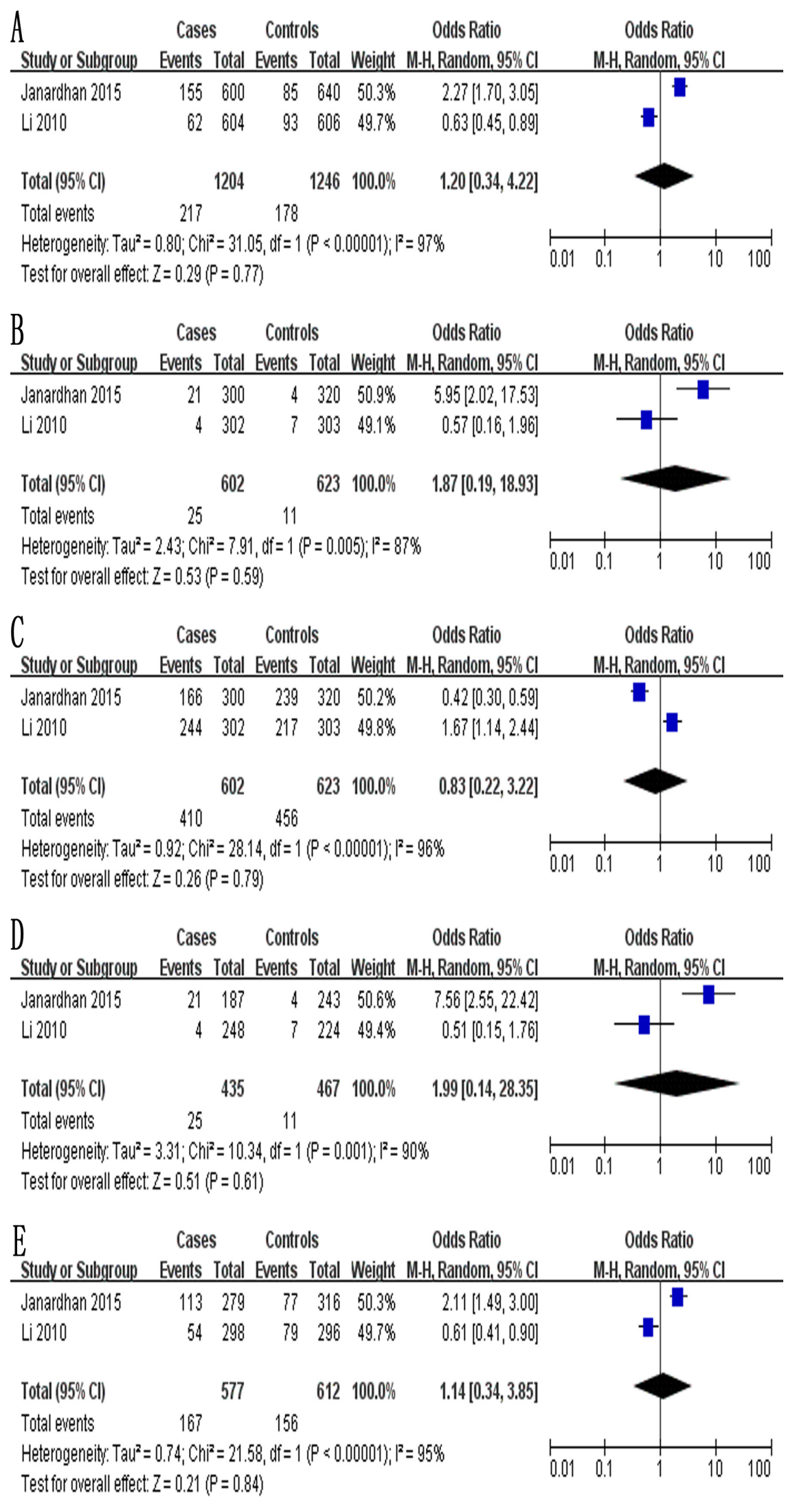

Figure 5: Forest plot describing the association between the -1154G/A polymorphism (rs1570360) and risk of ovarian cancer across all study participants according to five genetic models. (A) allelic (A-allele vs. G-allele), (B) recessive (AA vs. $\mathrm{GC}+\mathrm{GG}),(\mathbf{C})$ dominant (GG vs. GA + AA), (D) homozygous (AA vs. GG) and (E) heterozygous (GA vs. GG). 
control design was used to assess the association of at least one of the four target polymorphisms with ovarian cancer risk in humans; (2) full text was available, and sufficient data were reported to estimate an odds ratio (OR) with 95\% confidence interval (CI); and (3) genotype frequencies were reported. If multiple publications from the same research group appeared to report data for the same cases and controls, we included only the most recent publication in our meta-analysis.

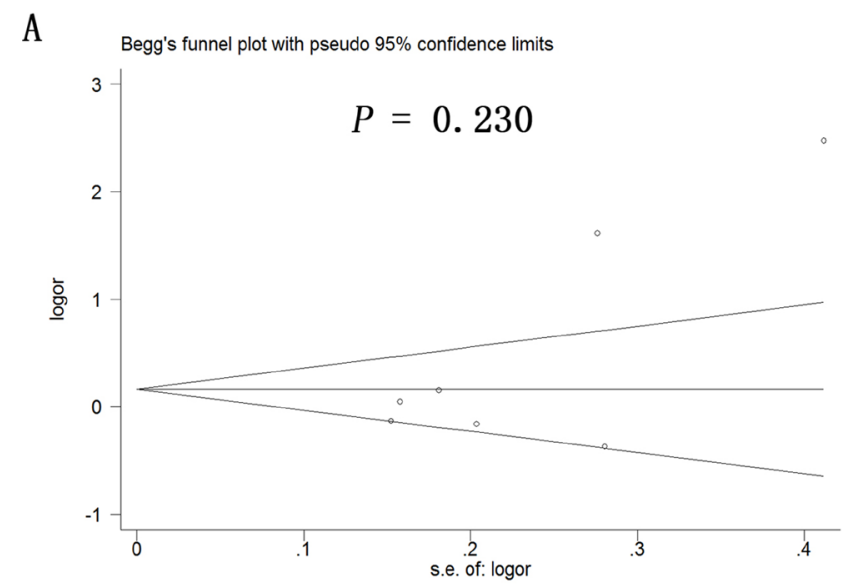

B
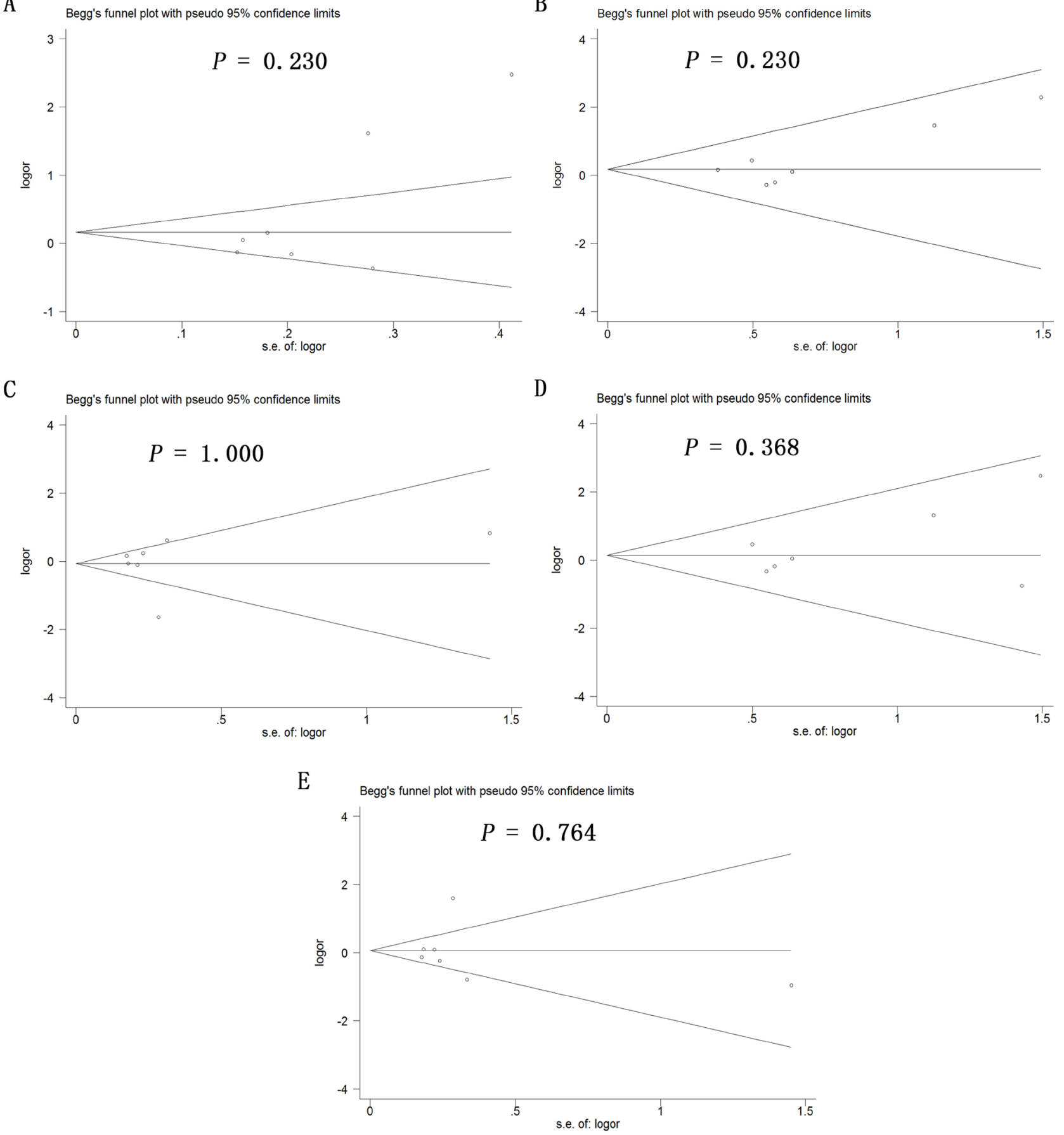

Figure 6: Begg's funnel plot to assess publication bias in the meta-analysis of a potential association between the $+936 \mathrm{C} / \mathrm{T}$ polymorphism (rs3025039) and risk of ovarian cancer across all study participants according to five genetic models. (A) allelic (T-allele vs. C-allele), (B) recessive (TT vs. CT + CC), (C) dominant (CC vs. CT + TT), (D) homozygous (TT vs. CC) and (E) heterozygous (CT vs. CC). 


\section{Data extraction}

Two authors (CHX and $\mathrm{ZHH}$ ) independently extracted the following data from included studies: first author's family name, year of publication, ethnicity, country of origin, testing methods, type of controls, $P$ value for HWE in controls, numbers and genotypes of cases and controls, frequencies of genotypes in cases and controls. Discrepancies were resolved by consensus.

\section{Assessment of methodological quality}

The quality of the included studies was assessed independently by two authors (CHX and $\mathrm{ZHH}$ ) according to the Newcastle-Ottawa Scale [35]. This scale awards
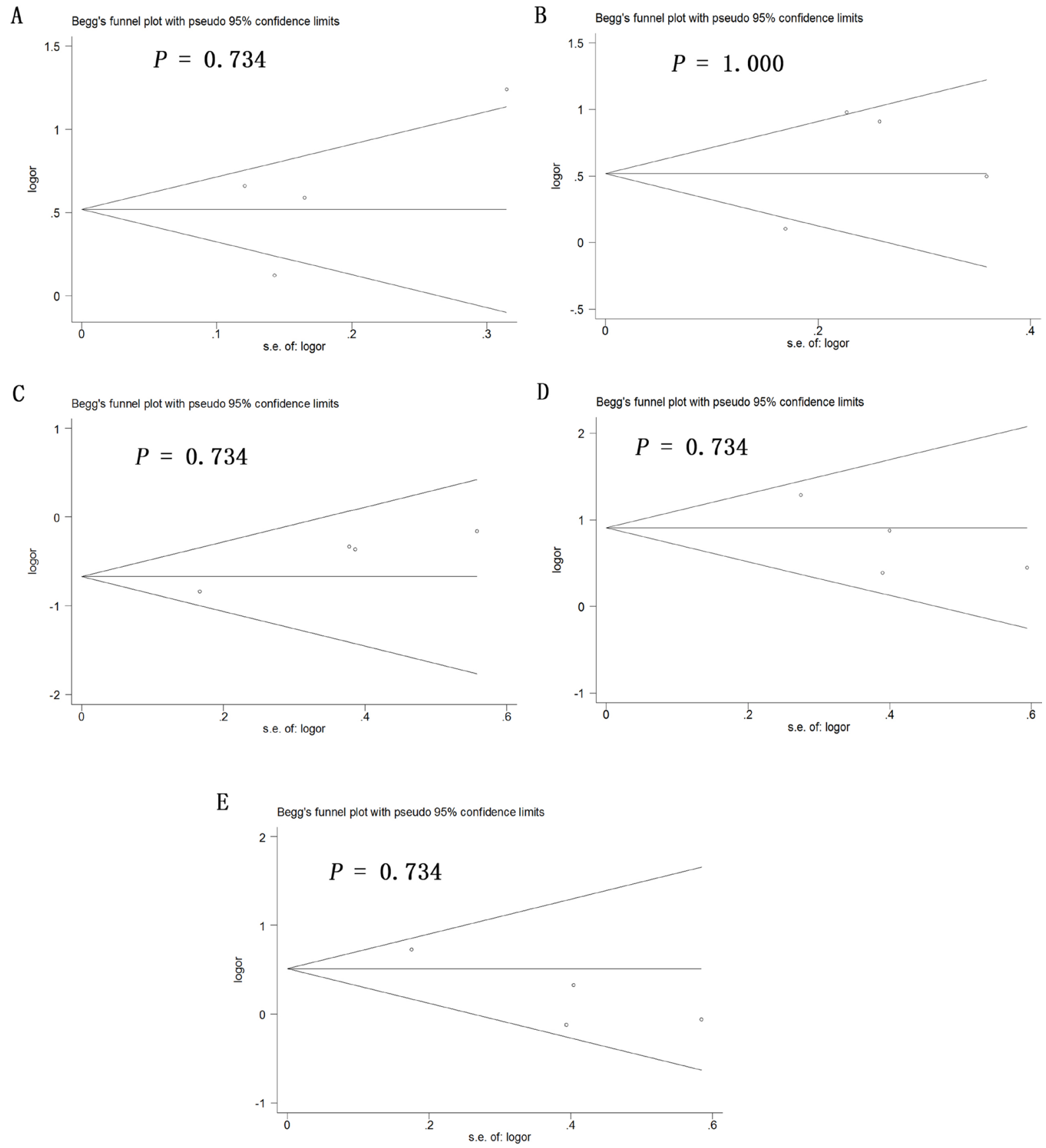

Figure 7: Begg's funnel plot to assess publication bias in the meta-analysis of a potential association between the $-460 \mathrm{C} / \mathrm{T}$ polymorphism (rs833061) and risk of ovarian cancer across all study participants according to five genetic models. (A) allelic (T-allele vs. C-allele), (B) recessive (TT vs. CT + CC), (C) dominant (CC vs. CT + TT), (D) homozygous (TT vs. CC) and (E) heterozygous (CT vs. CC). 
a maximum of 9 points to a study, with higher scores indicating better quality. Differences in quality score outcomes between the two assessors were solved by consensus. If consensus was not reached, a third assessor (HX) was consulted for the final decision.

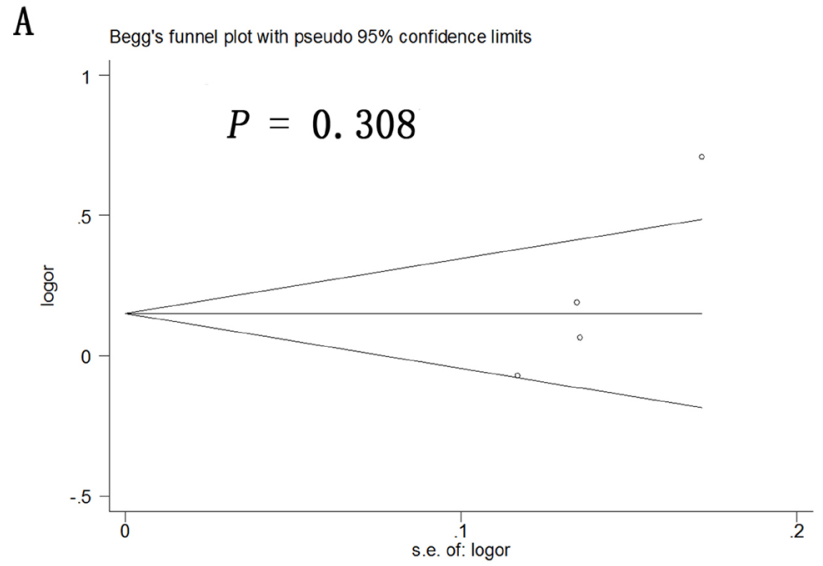

B
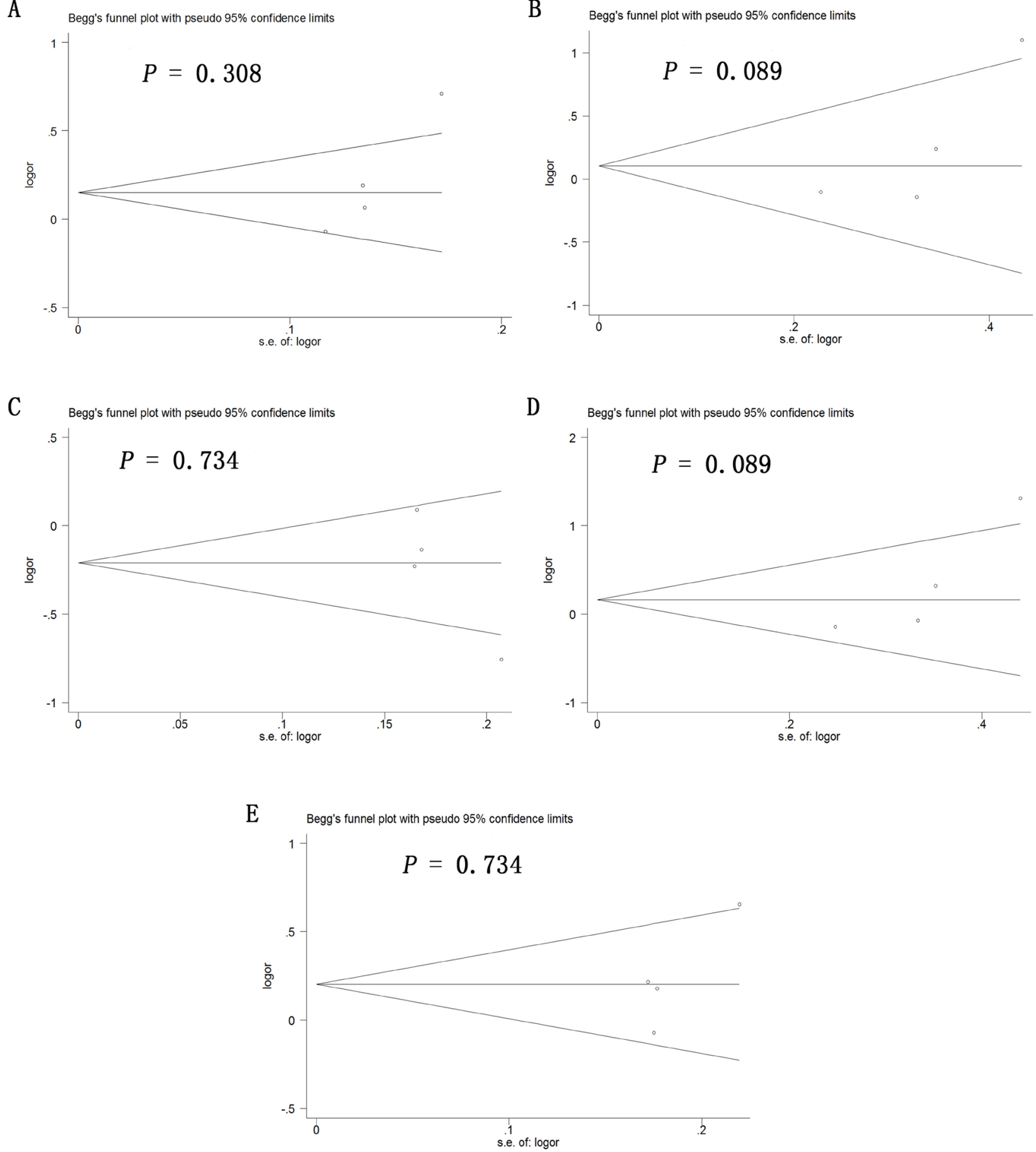

Figure 8: Begg's funnel plot to assess publication bias in the meta-analysis of a potential association between the -2578C/A polymorphism (rs699947) and risk of ovarian cancer across all study participants according to five genetic models. (A) allelic (A-allele vs. C-allele), (B) recessive (AA vs. CA + CC), (C) dominant (CC vs. CA + AA), (D) homozygous (AA vs. $\mathrm{CC})$ and (E) heterozygous (CA vs. CC). 
with ovarian cancer risk, based on genotype frequencies in cases and controls. The significance of pooled ORs was determined using the $Z$ test, with $P<0.05$ defined as the significance threshold. Meta-analysis was conducted using a fixed-effect model when $P>0.10$ for the $Q$ test, indicating lack of heterogeneity among studies; otherwise, a random-effect model was used. All statistical tests for meta-analyses were performed using Review Manager 5.2 (Cochrane Collaboration). Publication bias was assessed using Begg's funnel plot Stata 14.0 (Stata Corp, College Station, TX, USA), with $P<0.05$ considered statistically significant.

\section{Author contributions}

Designed the study: Hong Xu and Chao-Huan Xu. Searched databases and collected full-text papers: ChaoHuan Xu. Extracted and analyzed the data: Chao-Huan Xu and Zhong-Hui He. Statistical analyses: Chao-Huan Xu and Zhong-Hui He. Wrote the manuscript: Chao-Huan Xu. All authors reviewed the manuscript.

\section{CONFLICTS OF INTEREST}

The authors declare no competing financial interests.

\section{REFERENCES}

1. Siegel RL, Miller KD, Jemal A. Cancer statistics, 2016. CA Cancer J Clin. 2016; 66:7-30.

2. Reid BM, Permuth JB, Sellers TA. Epidemiology of ovarian cancer: a review. Cancer Biol Med. 2017; 14:9.

3. Legge F, Ferrandina G, Salutari V, Scambia G. Biological characterization of ovarian cancer: prognostic and therapeutic implications. Ann Oncol. 2005; 16:95-101.

4. Coleman RL, Monk BJ, Sood AK, Herzog TJ. Latest research and treatment of advanced-stage epithelial ovarian cancer. Nat Rev Clin Oncol. 2013; 10:211-24.

5. Sundar S, Neal RD, Kehoe S. Diagnosis of ovarian cancer. BMJ. 2015; 351:h4443.

6. Gharwan H, Bunch KP, Annunziata CM. The role of reproductive hormones in epithelial ovarian carcinogenesis. Endocr Relat Cancer. 2015; 22:R339-R363.

7. Hunn J, Rodriguez GC. Ovarian cancer: etiology, risk factors, and epidemiology. Gynecol Endocrinol. 2012; 55:3-23.

8. Romero I, Bast RC Jr. Minireview: human ovarian cancer: biology, current management, and paths to personalizing therapy. Endocrinology. 2012; 153:1593-1602.

9. Sellers TA, Schildkraut JM, Pankratz VS, Vierkant RA, Fredericksen ZS, Olson JE, Cunningham J, Taylor W, Liebow M, McPherson C, Hartmann LC, Pal T, Adjei AA. Estrogen bioactivation, genetic polymorphisms, and ovarian cancer. Cancer Epidemiol Biomarkers Prev. 2005; $14: 2536-2543$.
10. Yancopoulos GD, Davis S, Gale NW, Rudge JS, Wiegand SJ, Holash J. Vascular-specific growth factors and blood vessel formation. Nature. 2000; 407:242-248.

11. Shi YP, Ferrara N. OncogenicrasFails to Restore anin VivoTumorigenic Phenotype in Embryonic Stem Cells Lacking Vascular Endothelial Growth Factor (VEGF). Biochem Biophys Res Commun. 1999; 254:480-483.

12. Hazelton DA, Hamilton TC. Vascular endothelial growth factor in ovarian cancer. Curr Oncol Rep. 1999; 1:59-63.

13. Ferrara N, Gerber HP. The role of vascular endothelial growth factor in angiogenesis. Acta Haematol. 2001; 106:148-156.

14. Brustmann H, Naudé S. Vascular endothelial growth factor expression in serous ovarian carcinoma: relationship with high mitotic activity and high FIGO stage. Gynecol Oncol. 2002; 84:47-52.

15. Santin AD, Hermonat PL, Ravaggi A, Cannon MJ, Pecorelli S, Parham GP. Secretion of vascular endothelial growth factor in ovarian cancer. Eur J Gynaecol Oncol. 1998; 20:177-181.

16. Ogawa S, Kaku T, Kobayashi H, Hirakawa T, Ohishi Y, Kinukawa N, Nakano H. Prognostic significance of microvessel density, vascular cuffing and vascular endothelial growth factor expression in ovarian carcinoma: a special review for clear cell adenocarcinoma. Cancer Lett. 2002; 176:111-118.

17. Li L, Wang L, Zhang W, Tang B, Zhang J, Song H, Yao D, Tang Y, Chen X, Yang Z, Wang G, Li X, Zhao J, et al. Correlation of serum VEGF levels with clinical stage, therapy efficacy, tumor metastasis and patient survival in ovarian cancer. Anticancer Res. 2004; 24:1973-1979.

18. Hefler LA, Zeillinger R, Grimm C, Sood AK, Cheng WF, Gadducci A, Tempfer CB, Reinthaller A. Preoperative serum vascular endothelial growth factor as a prognostic parameter in ovarian cancer. Gynecol Oncol. 2006; 103:512-517.

19. Steffensen KD, Waldstrøm M, Brandslund I, Jakobsen A. The relationship of VEGF polymorphisms with serum VEGF levels and progression-free survival in patients with epithelial ovarian cancer. Gynecol Oncol. 2010; 117: 109-116.

20. Lose F, Nagle CM, O'Mara T, Batra J, Bolton KL, Song H, Ramus SJ, Gentry-Maharaj A, Menon U, Gayther SA, Pharoah PD, Kedda MA, Spurdle AB. Vascular endothelial growth factor gene polymorphisms and ovarian cancer survival. Gynecol Oncol. 2010; 119:479-483.

21. Yu L, Deng L, Li J, Zhang Y, Hu L. The prognostic value of vascular endothelial growth factor in ovarian cancer: a systematic review and meta-analysis. Gynecol Oncol. 2013; 128:391-396.

22. Hoeben A, Landuyt B, Highley MS, Wildiers H, Van Oosterom AT, De Bruijn EA. Vascular endothelial growth factor and angiogenesis. Pharmacol Rev. 2004; 56:549-580.

23. Watson CJ, Webb NJ, Bottomley MJ, Brenchley PE. Identification of polymorphisms within the vascular 
endothelial growth factor (VEGF) gene: correlation with variation in VEGF protein production. Cytokine. 2000; 12:1232-1235.

24. Yang B, Cross DF, Ollerenshaw M, Millward BA, Demaine AG. Polymorphisms of the vascular endothelial growth factor and susceptibility to diabetic microvascular complications in patients with type 1 diabetes mellitus. J Diabetes Complications. 2003; 17:1-6.

25. Mohammadi M, Ollier WER, Hutchinson IV. A functional association study of VEGF gene promoter polymorphisms with VEGF expression by stimulated pbm cells. Hum Immuno. 2003; 64:S125.

26. Konac E, Onen HI, Metindir J, Alp E, Biri AA, Ekmekci A. Lack of association between $-460 \mathrm{C} / \mathrm{T}$ and $936 \mathrm{C} / \mathrm{T}$ of the vascular endothelial growth factor and angiopoietin-2 exon $4 \mathrm{G} / \mathrm{A}$ polymorphisms and ovarian, cervical, and endometrial cancers. DNA Cell Biol. 2007; 26:453-463.

27. Jakubowska A, Gronwald J, Menkiszak J, Górski B, Huzarski T, Byrski T, Edler L, Lubiński J, Scott RJ, Hamann U. The VEGF_936_C > T 3' UTR polymorphism reduces BRCA1-associated breast cancer risk in Polish women. Cancer Lett. 2008; 262:71-76.

28. Jia JH. Association of Single Nucleotide Polymorphisms in the VEGF, MMP Genes with Epithelial Ovarian Carcinoma [dissertation]. Shijiazhuang, China: Hebei Medical University; 2009. [Article in Chinese].

29. Li Y, Wang Y, Kang S, Wang N, Zhou RM, Duan YN, Sun DL, Qin JJ, Zhao W, Zhao L. Association of vascular endothelial growth factor gene polymorphisms with susceptibility to epithelial ovarian cancer. Int J Gynecol Cancer. 2010; 20:717-723.
30. Rinck-Junior JA, Oliveira C, Lourenço GJ, Sagarra RA, Derchain SF, Segalla JG, Lima CS. Vascular endothelial growth factor (VEGF) polymorphism and increased risk of epithelial ovarian cancer. J Cancer Res Clin Oncol. 2015; 141:69-73.

31. Janardhan B, Vaderhobli S, Bhagat R, Chennagiri Srinivasamurthy P, Venketeshiah Reddihalli P, Gawari R, Krishnamoorthy L. Investigating impact of vascular endothelial growth factor polymorphisms in epithelial ovarian cancers: a study in the Indian population. PloS One. 2015; 10:e131190.

32. Zhang M, Cheng S, Ma Y, Qiao Y. Role of VEGF$2578 \mathrm{C} / \mathrm{A},+936 \mathrm{C} / \mathrm{T}$ and $-460 \mathrm{~T} / \mathrm{C}$ genetic polymorphisms in the risk of ovarian cancer in Chinese women. Int J Clin Exp Med. 2016; 9:18764-18771.

33. Zhang X, Qin J, Qin A. Three polymorphisms of vascular endothelial growth factor $(+936 \mathrm{C}>\mathrm{T},-460 \mathrm{C}>\mathrm{T}$, and$2578 \mathrm{C}>\mathrm{A})$ and their susceptibility to ovarian cancer: a meta-analysis. Int J Gynecol Cancer. 2015; 25: 779-785.

34. Moher D, Liberati A, Tetzlaff J, Altman DG; PRISMA Group. Preferred Reporting Items for Systematic Reviews and Meta-Analyses: The PRISMA Statement. PLoS Med. 2009; 6:e1000097.

35. Wells G A, Shea B, O'Connell D, Peterson J, Welch V, Losos M, Tugwell P. The Newcastle-Ottawa Scale (NOS) for assessing the quality of nonrandomised studies in meta-analyses. Available at: http://www.ohri.ca/programs/ clinical_epidemiology/oxford.htm. 